Notre Dame Journal of Formal Logic

Volume 42, Number 3, 2001

\title{
Four-valued Logic
}

\author{
Katalin Bimbó and J. Michael Dunn
}

\begin{abstract}
Four-valued semantics proved useful in many contexts from relevance logics to reasoning about computers. We extend this approach further. A sequent calculus is defined with logical connectives conjunction and disjunction that do not distribute over each other. We give a sound and complete semantics for this system and formulate the same logic as a tableaux system. Intensional conjunction (fusion) and its residuals (implications) can be added to the sequent calculus straightforwardly. We extend a simplified version of the earlier semantics for this system and prove soundness and completeness. Then, with some modifications to this semantics, we arrive at a mathematically elegant yet powerful semantics that we call generalized Kripke semantics.
\end{abstract}

\section{Introduction}

One might think that to know the world it is sufficient to know what are the truths. We disagree: one also needs to know what are the falsities. Of course, if one believes that if a proposition $p$ is not true then $p$ is false, then having been told that $p$ is not true, there is no need to be told that $p$ is false. This means-together with its contraposition, that is, that the nonfalsity of a proposition implies its truth-that any proposition is at least true or false. Accepting this principle does not exclude the possibility that a proposition has more than a single truth value. It might be controversial that a proposition can be both true and false in a truly ontological sense, but undoubtedly it can be doubly valued epistemically. The simplest example comes from ordinary language usage when one answers a yes-or-no question by "Yes and no." Colliding multiple entries in a database and inconsistent scientific theories are further illustrations of this phenomenon.

Classical logic assumes that each proposition has exactly one of the two truth values, 'true' and 'false'. Logics which allow propositions not to have truth values but to have "gaps" were called partial logics; their siblings, logics requiring propositions to

Received September 27, 2001; accepted December 21, 2002; printed August 29, 2003 2001 Mathematics Subject Classification: Primary, 03B47

Keywords: substructural logics, Lambek calculi, relevance logic, lattice representation, Kripke semantics

(C)2003 University of Notre Dame 
be at least true or at least false, possibly having "gluts," were less favored. The combination of these relaxations of the principle of bivalence leads to four possibilities, four new truth values.

The aim of this paper is to extend the four-valued approach to a large class of logics. Such an approach was started by Dunn's "proposition surrogates" and "coupled trees" ([9], [8]), and by Belnap's "useful four-valued logic" [3], and continued by others.

First we outline a basic sequent calculus together with a (sound and complete) semantics based on four values. Then we introduce an analytic tableaux formulation and prove that it is equivalent to our sequent calculus. Further, we add fusion and implications to the sequent calculus and to the semantics; we prove soundness and completeness. Lastly, we define a generalized Kripke semantics that is more elegant than the other semantics, and we prove soundness and completeness.

\section{Sequent Calculus and Situation Semantics}

A sequent calculus characterizes logical connectives by providing left and right introduction rules for them. ${ }^{1}$ Our basic logic $L N$ contains conjunction and disjunction as its logical connectives. A difference between $L N$ and the classical sequent calculus is that the comma is a binary structural connective in the former (',' is polyadic in the latter). We use the notation ' $\Gamma[\varphi]$ ' to single out a particular occurrence of the formula $\varphi$ in the structure $\Gamma$. In $L N$ only sequents of the form $\varphi \vdash \psi$ are provable, where $\varphi$ and $\psi$ are formulas; however, formulating the rules as below will allow us to extend $L N$ in Section 4 without changing these rules. $L N$ is defined as follows:

$$
\begin{aligned}
& \varphi \vdash \varphi \\
& \frac{\Gamma[\varphi] \vdash \chi}{\Gamma[\varphi \wedge \psi] \vdash \chi} \quad \frac{\Gamma[\psi] \vdash \chi}{\Gamma[\varphi \wedge \psi] \vdash \chi} \quad \frac{\Gamma \vdash \varphi \quad \Gamma \vdash \psi}{\Gamma \vdash \varphi \wedge \psi} \\
& \frac{\Gamma[\varphi] \vdash \chi \quad \Gamma[\psi] \vdash \chi}{\Gamma[\varphi \vee \psi] \vdash \chi} \quad \frac{\Gamma \vdash \varphi}{\Gamma \vdash \varphi \vee \psi} \quad \frac{\Gamma \vdash \psi}{\Gamma \vdash \varphi \vee \psi}
\end{aligned}
$$

In many logics, $\wedge$ and $\vee$ distribute over each other-this makes it easy to provide semantics for such systems (cf. Belnap [2]). With a little fiddling one can check that neither $\varphi \wedge(\psi \vee \chi) \vdash(\varphi \wedge \psi) \vee \chi$ nor $\varphi \wedge(\psi \vee \chi) \vdash(\varphi \wedge \chi) \vee(\varphi \wedge \chi)$ is provable in $L N$, since we did not postulate any structural rules. The fact that the introduction rules for these connectives do not lead to distributivity shows that nondistributive logics are perfectly natural.

Before we proceed to defining the semantics for $L N$ we recall the four-valued clauses for $\wedge$ and $\vee$ from [8] (where those are given for the first-degree entailment).

$$
\begin{array}{lllll}
t \in|\varphi \wedge \psi| & \text { iff } & t \in|\varphi| & \text { and } & t \in|\psi|, \\
f \in|\varphi \wedge \psi| & \text { iff } & f \in|\varphi| \text { or } & f \in|\psi|, \\
t \in|\varphi \vee \psi| & \text { iff } & t \in|\varphi| & \text { or } & t \in|\psi|, \\
f \in|\varphi \vee \psi| & \text { iff } & f \in|\varphi| \text { and } & f \in|\psi| .
\end{array}
$$


The function || assigns a set of truth values to formulas, and ' $t \in$ ' and ' $f \in$ ' are read as 'at least true' and 'at least false'. We cannot assign values in a semantics for $L N$ in this way, since according to (1) and (2), and the ordinary usage of 'and' and 'or' in the metalanguage, if $\varphi \wedge(\psi \vee \chi)$ is at least true, so is $(\varphi \wedge \psi) \vee(\varphi \wedge \chi)$. In our intuitive semantics we weaken (1) and (2) to (3) and (4):

$$
\begin{array}{rll}
t \in|\varphi \wedge \psi| & \text { iff } & t \in|\varphi| \text { and } t \in|\psi|, \\
f \in|\varphi \wedge \psi| & \text { iff } & \text { not both } t \in|\varphi| \text { and } t \in|\psi|, \\
t \in|\varphi \vee \psi| & \text { iff } & \text { not both } f \in|\varphi| \text { and } f \in|\psi|, \\
f \in|\varphi \vee \psi| & \text { iff } & f \in|\varphi| \text { and } f \in \mid \psi .
\end{array}
$$

One could view the new clauses as merely rewriting the old ones using De Morgan laws; however, our intuitions go deeper than that. We start with the idea that the truth condition for conjunction is clearer than its falsity condition, whereas the opposite is true of disjunction. We add to this the further idea that 'at least true' and 'at least false' should be linked by something like a negation. Clearly, this negation cannot be classical, that is, we do not assume that (3) - (4) are equivalent to (1) - (2).

A "usual" possible world—as in classical modal logics—cannot make a sentence true and false at once. Thus, instead of an ordinary possible world we think of a pair $\left\langle w^{+}, w^{-}\right\rangle=w$, a positive and a negative situation put together, as one characterizing a state of affairs. We shall call $w$ a situation. Positive and negative situations are logically closed sets of sentences, "the truths" and "the falsities" (of course, the last is logically closed in the reverse direction as we shall see). We say that $\varphi$ is (at least) true if and only if $\varphi \in w^{+}$, and $\varphi$ is (at least) false if and only if $\varphi \in w^{-}$.

The semantics for $L N$ is defined on a frame $\mathcal{F}$ consisting of two sets, the set of positive situations $W^{+}$and the set of negative situations $W^{-}$, and a binary relation $Q$ between them (i.e., $Q \subseteq W^{+} \times W^{-}$). ${ }^{2} \quad$ We define two functions, $\ell$ and $r$, on subsets of the $W \mathrm{~s}$ as

$$
\begin{aligned}
& r(X)=\left\{y \in W^{-}:(\forall x \in X)(x Q y)\right\}, \\
& \ell(Y)=\left\{x \in W^{+}:(\forall y \in Y)(x Q y)\right\} .
\end{aligned}
$$

A model $\mathcal{M}$ is a frame augmented with a valuation function $v$, a function from the set of propositional variables into $\wp\left(W^{+}\right) \times \wp\left(W^{-}\right)$such that

$$
\text { if } v(p)=\langle X, Y\rangle \text {, then } r(X)=Y \text { and } \ell(Y)=X \text {. }
$$

To keep our notation transparent we use $v^{+}(\varphi)$ and $v^{-}(\varphi)$ to refer to the first and the second member of $v(\varphi)$. We also postulate that, in a model, the domain of the valuation function can be extended for complex formulas by the recursive clauses,

$$
\begin{aligned}
& v(\varphi \wedge \psi)=\left\langle v^{+}(\varphi) \cap v^{+}(\psi), r\left(v^{+}(\varphi) \cap v^{+}(\psi)\right)\right\rangle, \\
& v(\varphi \vee \psi)=\left\langle\ell\left(v^{-}(\varphi) \cap v^{-}(\psi)\right), v^{-}(\varphi) \cap v^{-}(\psi)\right\rangle,
\end{aligned}
$$

so that both $\ell v^{-}(\varphi \wedge \psi)=v^{+}(\varphi \wedge \psi)$ and $r v^{+}(\varphi \vee \psi)=v^{-}(\varphi \vee \psi)$.

We note that over the set $\{\langle X, Y\rangle: r(X)=Y$ and $\ell(Y)=X\}$ any $v$ satisfying (6) can be extended according to (7)-(8) (cf. Birkhoff [5], 5§7). The next lemma captures the interaction of the functions $r, \ell$ and of the valuation function $v$ in a model. 
Lemma $2.1\left(\ell, \boldsymbol{r}\right.$ and $\left.v^{+}, \boldsymbol{v}^{-}\right) \quad$ If $v(\varphi)=\langle X, Y\rangle$ in a model $\mathcal{M}$, then the following hold:

1. $r v^{+}(\varphi)=r X=Y=v^{-}(\varphi)$,

2. $\ell v^{-}(\varphi)=\ell Y=X=v^{+}(\varphi)$,

3. $\ell r X=X$ and $r \ell Y=Y$.

Proof This lemma is easily provable from the definition of the valuation.

We will use the notation $w \vDash \varphi$ (where $w=\left\langle w^{+}, w^{-}\right\rangle$) to indicate that ' $\varphi$ is (at least) true in the situation $w^{\prime}$. In other words, $w \vDash \varphi$ if and only if $w^{+} \in v^{+}(\varphi)$. Furthermore, $\varphi \vDash \psi$ is to mean that for any $w$, if $w \vDash \varphi$ then $w \vDash \psi$. Provided all the definitions, we have a model for the logic $L N$-as stated in the following theorem.

Theorem 2.2 (Soundness) If $\varphi \vdash \psi$ then $\varphi \vDash \psi$.

Proof The structure of the proof is as usual; we consider only one of the cases beyond noting that the axiom scheme is easily seen to be sound. Assume that $\chi \vDash \varphi$, $\chi \vDash \psi$ and $w \vDash \chi$, that is, $w^{+} \in v^{+}(\chi)$. By the assumptions $w^{+} \in v^{+}(\varphi)$ and $w^{+} \in v^{+}(\psi)$; hence $w^{+} \in v^{+}(\varphi) \cap v^{+}(\psi)$, that is, $w^{+} \in v^{+}(\varphi \wedge \psi)$ as desired.

Before defining the canonical frame and the canonical model we accumulate further definitions and lemmas. Recall that a set of sentences $T$ is a theory if and only if

(a) $T$ is closed under consequence, that is, if $\varphi \in T$ and $\varphi \vdash \psi$ then $\psi \in T$, and

(b) $T$ is closed under conjunction, that is, $\varphi, \psi \in T$ only if $\varphi \wedge \psi \in T$.

We shall call such theories positive, and in addition we define negative theories. ${ }^{3}$ A set of sentences $T$ is a negative theory if and only if

(a) $T$ is backward closed under logical consequence, that is, if $\psi \in T$ and $\varphi \vdash \psi$, then $\varphi \in T$, and

(b) $T$ is closed under disjunction, that is, $\varphi, \psi \in T$ only if $\varphi \vee \psi \in T$.

These definitions harmonize with the intuitions we offered in support of (3)-(4) and with what is reasonable to expect about the interaction of logical consequence with truth and falsity. A theory, a positive or a negative one, is called inherent if and only if it cannot be obtained from two other theories (respectively, positive or negative ones) by taking the sentences common to both. Extending sets of sentences to theories is particularly useful, since theories have the above closure properties.

Lemma 2.3 (Extension to theories) If $\gamma_{0} \wedge \cdots \wedge \gamma_{n-1} \nvdash \varphi$ for arbitrary finite $\left\{\gamma_{0}, \ldots, \gamma_{n-1}\right\} \subseteq \Gamma$ then there is a positive theory, denoted as $\mathrm{Th}^{+}(\Gamma)$ which contains $\Gamma$ but does not contain $\varphi$. Similarly, provided $\varphi \nvdash \gamma_{0} \vee \cdots \vee \gamma_{n-1}$ there is a negative theory $\mathrm{Th}^{-}(\Gamma)$ containing $\Gamma$ but not containing $\varphi$.

Proof The proof of the first half of the lemma is standard; the second half is provable by dualizing the argument.

The next lemma states that consistent sets of sentences can be maximalized with respect to not containing a certain sentence; moreover, the resulting theory is inherent. Here inherent theories play the role that maximally consistent theories do in a Henkin-style completeness proof for classical logic. ${ }^{4}$

Lemma 2.4 (Separation by an inherent theory) If $\Gamma \nvdash \varphi(\varphi \nvdash \Gamma)$, then there is a positive (negative) inherent theory $T$ containing $\Gamma$ but not containing $\varphi$. 
Proof One has to prove separately the case when $T$ is positive and when $T$ is negative. We provide some of the details of the first case; the second part is, mutatis mutandis, obvious.

Let us assume that all the formulas are enumerated in a list as $\delta_{0}, \ldots, \delta_{n-1}, \ldots$. We define a series of theories inductively as

1. $T_{0}=\mathrm{Th}^{+}(\Gamma)$,

$$
\text { 2. } T_{n+1}= \begin{cases}\mathrm{Th}^{+}\left(T_{n} \cup\left\{\delta_{n+1}\right\}\right) & \text { if } \bigwedge \mathrm{Th}^{+}\left(T_{n} \cup\left\{\delta_{n+1}\right\}\right) \nvdash \varphi, \\ T_{n} & \text { otherwise. }\end{cases}
$$

By ' $\wedge X \vdash y$ ' we mean that for some finite subset $\left\{x_{0}, \ldots, x_{m-1}\right\}$ of $X$, $x_{0} \wedge \cdots \wedge x_{m-1} \vdash y$. Let $T$ be the union of these theories. Certainly, $\Gamma \subseteq T$ and $\varphi \notin T$ by the construction. To show that $T$ is inherent, let us assume that it is not. Then there are two other theories $T^{\prime}$ and $T^{\prime \prime}$ which both contain $T$. That is, there are two sentences $\psi$ and $\chi$ such that $\psi \in T^{\prime}$ but $\psi \notin T^{\prime \prime}$ and $\chi \in T^{\prime \prime}$ but $\chi \notin T^{\prime}$; therefore $\psi, \chi \notin T$. However, then there are conjunctions $\xi_{l}$ (from $T^{\prime}$ ) and $\xi_{k}$ (from $T^{\prime \prime}$ ) such that $\xi_{l} \wedge \psi \vdash \varphi$ and $\xi_{k} \wedge \chi \vdash \varphi$. Then $\varphi \in T^{\prime}, T^{\prime \prime}$ contradicting $\varphi \notin T$.

Now we can proceed to define the canonical frame and the canonical model. The canonical frame consists of the set of positive inherent theories $\mathcal{T}^{+}$, of the set of negative inherent theories $\mathcal{T}^{-}$, and of the relation $Q$ which is nonempty intersection between theories and will be denoted by $\ell$. The canonical interpretation function on the propositional variables is defined as

$$
v(p)=\left\langle\left\{T^{+} \in \mathcal{T}^{+}: p \in T^{+}\right\},\left\{T^{-} \in \mathcal{T}^{-}: p \in T^{-}\right\}\right\rangle .
$$

We prove that the canonical model is a model defined on a frame as defined above, and in addition it has the separation property. In other words, $L N$ is complete with respect to the canonical model.

Theorem 2.5 (Completeness) If $\varphi \vDash \psi$ then $\varphi \vdash \psi$.

Proof The canonical frame is a frame, since $\ell r$ and $r \ell$ are so-called closure operators. Therefore, the set of $\ell r$-closed subsets of $\mathcal{T}^{+}$and the set of $r \ell$-closed subsets of $\mathcal{T}^{-}$are closed under intersection (cf. [5]). Thus, any valuation satisfying condition (6) can be extended according to (7) - (8). In particular, the canonical valuation is such that $r v^{+}(p)=v^{-}(p)$ and $\ell v^{-}(p)=v^{+}(p)$.

(1) As usual, we prove the contrapositive of the claim stated in the completeness theorem. Thus, assume that $\varphi \nvdash \psi$. According to Lemma 2.4, there is an inherent theory that extends $\{\varphi\}$ but does not contain $\psi$. Let us denote this theory by $T_{\varphi}^{+}$. As a negative theory we take an inherent theory $T_{\varphi}^{-}$which extends $\operatorname{Th}^{-}(\{\varphi\})$; there is always such a negative theory. Let $w=\left\langle T_{\varphi}^{+}, T_{\varphi}^{-}\right\rangle$. Then clearly $w \vDash \varphi$ but $w \not \models \psi-$ provided that $v(\varphi)=\left\langle\left\{T^{+}: \varphi \in T^{+}\right\},\left\{T^{-}: \varphi \in T^{-}\right\}\right\rangle$for arbitrary $\varphi$. We prove now, by induction on the structure of formulas, that given the canonical valuation $v$, this is indeed the case.

(2) As for conjunction, assume that $T^{+} \in v^{+}(\varphi \wedge \psi)$. The following series of iffs holds (for reasons indicated in the brackets): 


$$
\begin{array}{rlrl}
T^{+} \in v^{+}(\varphi \wedge \psi) & \Leftrightarrow T^{+} \in v^{+}(\varphi) \cap v^{+}(\psi) & & \text { [def. of } v \text { ] } \\
& \Leftrightarrow T^{+} \in v^{+}(\varphi) \& T^{+} \in v^{+}(\psi) & & \text { [def. of } \cap \text { ] } \\
& \Leftrightarrow \varphi \in T^{+} \& \psi \in T^{+} & & \text {[Ind. hyp.] } \\
& \Leftrightarrow \varphi \wedge \psi \in T^{+} & & \text {[def. of } T^{+} \text {] } \\
T^{-} \in v^{-}(\varphi \wedge \psi) & \Leftrightarrow T^{-} \in r\left(v^{+}(\varphi) \cap v^{+}(\psi)\right) & & \text { [def. of } v \text { ] } \\
& \Leftrightarrow \forall T^{+}\left(T^{+} \in v^{+}(\varphi) \cap v^{+}(\psi) \Rightarrow T^{+} \gamma T^{-}\right) & \text {[def. of } r \text { ] } \\
& \Leftrightarrow \forall T^{+}\left(\varphi \in T^{+} \& \psi \in T^{+} \Rightarrow T^{+} \gamma T^{-}\right) & & \text {[def. of } \cap, \\
& \Leftrightarrow \varphi \wedge \psi \in T^{-} & & \text {IH] } \\
& & & \text { [def. of } T \text { s] }
\end{array}
$$

(3) The case of disjunction is the dual of case (2).

\section{Analytic Tableaux}

An analytic tableau is an alternative, frequently a very elegant formulation for a logic (cf. Smullyan [18] and [9]). Since $L N$ is a positive logic we will opt for building a tableau as a pair of trees rather than a single tree. Certainly we cannot simply adopt the analytic tableaux for classical logic or the so-called coupled trees for first-degree entailment. Our intuition is that the lack of distributivity, in case of trees, means that while we want to keep track of all the subformulas of a formula, the notion of a branch in a tree cannot be defined to build in any structural rules, so to speak. In [9], since distribution can be proven, the structural rule of thinning is somehow built in into the trees. As we prove below, distribution is not provable in our system.

Informally speaking, to see whether $\varphi$ implies $\psi$ we build two trees, one with the root $\varphi$ (growing downward) and another with the root $\psi$ (growing upward). The rules for the two trees are different. The top tree is built by the following two rules:

$$
\begin{array}{ll}
\varphi \wedge \psi & \varphi \vee \psi \\
\varphi \| \psi & \varphi \mid \psi
\end{array}
$$

The bottom tree is built using similar rules, except $\|$ and | are interchanged. Note that $\|$ and | are both to be interpreted as a kind of branching; however, one could think of | as separating alternatives each of which should hold and of $\|$ as separating possible choices.

$$
\begin{aligned}
\varphi \mid \psi & \varphi \| \psi \\
\varphi \wedge \psi & \varphi \vee \psi
\end{aligned}
$$

Now we proceed to the formal definition of analytic tableaux.

Definition 3.1 (Analytic tableaux) An analytic tableau for $\varphi \vdash \psi$ is a pair consisting of a top tree $T_{t}$ and a bottom tree $T_{b} . T_{t}$ and $T_{b}$ are ordered dyadic trees with two types of forking, ${ }^{5}$ where the points of the tree are (occurrences of) formulas. The root of $T_{t}$ is $\varphi$; the root of $T_{b}$ is $\psi$. Assuming that the trees constructed up to a point are $T_{t}$ and $T_{b}$, the trees are recursively extended as follows:

(i) if there is a leaf in $T_{t}$ of the form $\varphi \wedge \psi$ or $\varphi \vee \psi$, then replace the leaf with an ordered binary tree according to the rules (9),

(ii) if there is a leaf in $T_{b}$ of the form $\varphi \wedge \psi$ or $\varphi \vee \psi$, then replace the leaf with an ordered binary tree according to the rules (10).

The analytic tableaux are complete when neither (i) nor (ii) is applicable. 
It can be seen easily that each analytic tableau is finite: formulas are of finite length, and each rule induces finite branching, where the leaves in the rules (9) and (10) are proper subformulas of the root formula. Furthermore, each leaf of both trees of a complete analytic tableau is a propositional variable. It is easy to see that the complete tableau for $\varphi \vdash \psi$ is unique. To define the logical consequence relation for analytic tableaux we need a notion of covering.

Definition 3.2 (Covering relation) Covering is a relation between formulas in the top tree and formulas in the bottom tree in an analytic tableau which holds in the following cases:

1. a propositional letter covers itself,

2. $\varphi$ covers $\chi$ or $\psi$ covers $\chi$ iff $\varphi \wedge \psi$ covers $\chi$, $\varphi$ covers $\chi$ and $\psi$ covers $\chi$ iff $\varphi \vee \psi$ covers $\chi$,

3. $\chi$ covers $\varphi$ and $\chi$ covers $\psi$ iff $\chi$ covers $\varphi \wedge \psi$, $\chi$ covers $\varphi$ or $\chi$ covers $\psi$ iff $\chi$ covers $\varphi \vee \psi$.

Now we can state formally the notion of consequence in analytic tableaux.

Definition 3.3 (Tableau proof) An analytic tableau $T$ proves that $\psi$ is a consequence of $\varphi$ (i.e., $\varphi \vdash \psi$ ) if and only if $T$ is the complete analytic tableau for $\varphi \vdash \psi$ and $\varphi$ covers $\psi$.

To illustrate the use of tableaux we give two nontrivial examples. First, we give the tableau proof of $(p \wedge q) \vee(p \wedge r) \vdash p \wedge(q \vee r)$. (In the two tableaux below, nodes are labeled by binary strings for easy reference.)

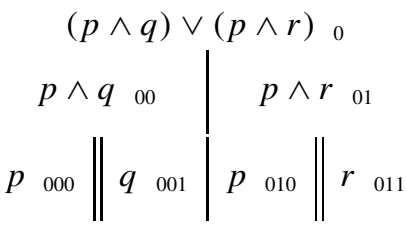

$$
\begin{aligned}
& \left.p_{00}\right|_{p \wedge(q \vee r)_{0}} ^{q} q \vee r_{01}
\end{aligned}
$$

The tableau is complete. Since $p_{000}$ covers $p_{00}$ and $q_{001}$ covers $q_{010}$, therefore, $q \vee r_{01}$, so we can conclude that $p \wedge q_{00}$ covers $p \wedge(q \vee r)_{0}$. We should verify that so does $p \wedge r_{01}$. That is, we should examine whether $p \wedge r_{01}$ covers both $p_{00}$ and $q \vee r_{01}$. The first is immediate: $p_{010}$ covers $p_{00}$. The second follows from $r_{011}$ covering $r_{011}$. This shows that $(p \wedge q) \vee(p \wedge r)$ covers $p \wedge(q \vee r)$. 
As an example for a disproof we take the converse of the above sequent: $p \wedge(q \vee r) \vdash(p \wedge q) \vee(p \wedge r)$.

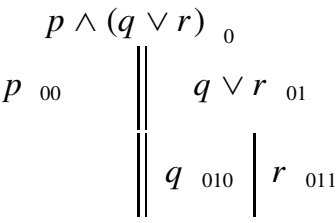

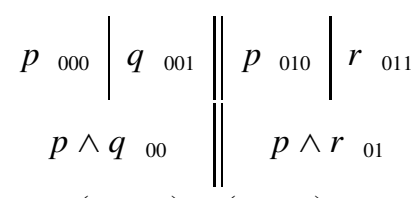

$$
\begin{aligned}
& (p \wedge q) \vee(p \wedge r)_{0}
\end{aligned}
$$

Again the analytic tableau is complete, and to see that it refutes the alleged consequence, it is sufficient to note first, that $p_{00}$ covers neither $q_{001}$ nor $r_{011}$ and second, that $q \vee r_{01}$ does not cover the $p$ s $\left(p_{000}, p_{010}\right)$.

These two examples support our intuition that the analytic tableaux we defined do capture the same consequence relation as $L N$ does. The next two theorems prove this intuition to be correct.

Theorem 3.4 (From tableaux to sequents) If $T$ is the complete analytic tableau for $\varphi \vdash \psi$ and $\varphi$ covers $\psi$, then $\varphi \vdash \psi$ is a theorem of $L N$.

Proof The proof is by straightforward induction on the structure of the tableau $T$. We go through only two of the cases.

Case 1 Assume that each of the two trees is a single propositional variable, and $p$ covers $p$. In $L N, p \vdash p$ is an instance of the axiom scheme.

Case 2 If the root of $T_{t}$ is a complex formula, then first let us assume that it is a conjunction. If $\varphi \wedge \psi$ covers $\chi$, then by definition $\varphi$ covers $\chi$ or $\psi$ covers $\chi$. By the hypothesis of induction $\varphi \vdash \chi$ or $\psi \vdash \chi$ is derivable in $L N$. In either case, we get $\varphi \wedge \psi \vdash \chi$ in a single step by an application of one of the two left introduction rules for $\wedge$.

The next theorem states the other direction of the equivalence.

Theorem 3.5 (From sequents to tableaux) If $\varphi \vdash \psi$ is provable in $L N$, then there is a tableau proof for $\varphi \vdash \psi$.

Proof The proof is by induction on the structure of derivations in $L N$. We include here three of the five cases.

Case $1 \varphi \vdash \varphi$ is the only axiom scheme, and so we show that for any formula $\varphi$, there is a tableau proof of $\varphi \vdash \varphi$. For propositional variables the claim holds trivially. Otherwise, $\varphi$ is either $\varphi_{1} \vee \varphi_{2}$ or $\varphi_{1} \wedge \varphi_{2}$, and so the complete tableaux for $\varphi \vdash \varphi$ look like the following: 
(i)

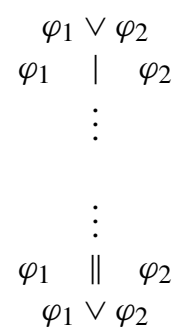

(ii)

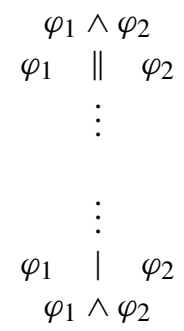

By inductive hypothesis the complete subtree for $\varphi_{1}$ (in $T_{t}$ ) covers the complete subtree for $\varphi_{1}$ (in $T_{b}$ ) and similarly for $\varphi_{2}$. Then, by the definition of the covering relation, $\varphi_{1} \vee \varphi_{2}$ covers $\varphi_{1} \vee \varphi_{2}$, and $\varphi_{1} \wedge \varphi_{2}$ covers $\varphi_{1} \wedge \varphi_{2}$.

Case 2 Assume that $\varphi \wedge \psi \vdash \chi$ is the last line of a derivation that is obtained from $\varphi \vdash \chi$ or $\psi \vdash \chi$ by an application of $(\wedge \vdash)$. Then, by the induction hypothesis, there is a tableau proof for the premise, let us say, for $\varphi \vdash \chi$. Construct the tableau proof for $\varphi \wedge \psi \vdash \chi$ as follows: take $T_{t}$ and add a new $\operatorname{root} \varphi \wedge \psi$ with $\|$ branching, where the two immediate subtrees are $T_{t}$ and the complete (top) tree for $\psi$ (this always exists and is unique as we argued before). By the definition of covering, $\varphi \wedge \psi$ covers $\chi$ in the new complete tableau for $\varphi \wedge \psi \vdash \chi$.

Case 3 Assume that the last line in a derivation in $L N$ is $\varphi \vee \psi \vdash \chi$ obtained by $(\vee \vdash)$. Then $\varphi \vdash \chi$ and $\psi \vdash \chi$ is the last but one line of the derivation. The inductive hypothesis ensures that there are complete tableaux for $\varphi \vdash \chi$ and $\psi \vdash \chi$, with appropriate covering. The complete tableau for $\varphi \vee \psi \vdash \chi$ is constructed as follows: $T_{t}$ has $\varphi \vee \psi$ as its root with the trees for $\varphi$ and $\psi$ as immediate subtrees joined by $\mid . T_{b}$ is one of the copies of the tree for $\chi$. Since both $\varphi$ and $\psi$ cover $\chi$, so does $\varphi \vee \psi$.

The two formalisms we presented are limited to relatively simple formulas that contain only conjunction and disjunction. In the next section we extend $L N$ and provide a sound and complete semantics.

\section{Adding Intensional Connectives}

Our aim in this section is to adapt the four-valued treatment to systems which have fusion and two implications beyond conjunction and disjunction. The sequent calculus can be extended straightforwardly to contain $\circ, \rightarrow$, or $\leftarrow$ (cf. Routley et al. [17], [8], [2]). As we noted earlier, structures are formed by pairing, and brackets indicate an occurrence of a structure in another structure. (No additional properties, as for example, associativity or commutativity, are supposed to be true of the comma to start with.) The following rules are added to $L N$ :

$$
\begin{array}{cc}
\frac{\Gamma[\varphi, \psi] \vdash \chi}{\Gamma[(\varphi \psi)] \vdash \chi} & \frac{\Gamma_{1} \vdash \varphi \Gamma_{2} \vdash \psi}{\Gamma_{1}, \Gamma_{2} \vdash(\varphi \circ \psi)} \\
\frac{\Gamma_{1} \vdash \varphi \quad \Gamma_{2}[\psi] \vdash \chi}{\Gamma_{2}\left[\Gamma_{1},(\varphi \rightarrow \psi)\right] \vdash \chi} & \frac{\varphi, \Gamma \vdash \psi}{\Gamma \vdash(\varphi \rightarrow \psi)} \\
\frac{\Gamma_{1} \vdash \varphi \Gamma_{2}[\psi] \vdash \chi}{\Gamma_{2}\left[(\psi \leftarrow \varphi), \Gamma_{1}\right] \vdash \chi} & \frac{\Gamma, \varphi \vdash \psi}{\Gamma \vdash(\psi \leftarrow \varphi)}
\end{array}
$$


Note that we still do not assume any structural rules in the system; nevertheless, there are certain properties which link the connectives. Fusion is lattice ordered (cf. Meyer and Routley [16]) and the implications are the residuals of the fusion. Such a logic can, arguably, be called the minimal substructural logic, and hence we denote the system by $L S$. Most of the ordinary assumptions concerning these connectives can be added in a standard fashion.

Formally, the following sequents correspond to the above properties, and their provability in $L S$ can be easily checked:

$$
\begin{gathered}
\varphi \circ(\psi \vee \chi) \dashv(\varphi \circ \psi) \vee(\varphi \circ \chi), \quad \text { and } \\
(\varphi \vee \psi) \circ \chi \dashv(\varphi \circ \chi) \vee(\psi \circ \chi), \\
\varphi \vdash \chi \leftarrow \psi \quad \text { iff } \quad \varphi \circ \psi \vdash \chi \quad \text { iff } \quad \psi \vdash \varphi \rightarrow \chi .
\end{gathered}
$$

The crucial question is how to define the truth and the falsity conditions for fusion and implications. In order to obtain a simplified semantics from that in Section 2, we will use theories instead of inherent theories in the canonical model. We note that now only Lemma 2.3 is needed for the separation property, and Theorem 2.5, that is, completeness, is provable as before-a brief inspection of the second part of the earlier proof reveals that it does not depend on theories being inherent.

The truth conditions for the intensional operators can be defined in the usual way (cf. Jónsson and Tarski [14, 15] and [16]). In fact, in Bimbó [4] the intensional operations are defined on the filters in the principal pairs. If one wants to characterize the falsity conditions of the intensional operations directly from a relation (and not only via the Galois functions), then the definitions of the frame and that of the operations get slightly more complicated.

The difficulty stems from the fact that the implications have mixed tonicity; moreover, when these operations are defined from a relation that represents $\circ$ on filters it is essential that $\rightarrow$ and $\leftarrow$ are the two residuals. We will use insights from Curry ([7], $4 \mathrm{C} 1$ ) to find appropriate relations that provide a definition of falsity for implications. The two implications can be thought of as the maximal solutions among all the solutions to the inequality $x \circ y \leq c$, solving it for $x$ and $y$, respectively. That is, the solutions form negative theories and the implications are at the top in these theories.

Accordingly, we add to the frame defined in Section 2 three relations, $R^{+}$, to define the truth of the intensional operations, and $R_{\rightarrow}^{-}, R_{\leftarrow}^{-}$, to define their falsity. $R^{+} \subseteq\left(W^{+}\right)^{3}$ as usual; however, $R_{\rightarrow}^{-} \subseteq W^{+} \times W^{-} \times W^{-}$and $R_{\leftarrow}^{-} \subseteq W^{+} \times W^{-} \times W^{-}$. The two latter relations connect positive and negative situations, and either of the two lends itself to a definition of the falsity of $\circ$ via residuation. Therefore, we postulate both of these definitions under (13) with the proviso that $v^{-}(\varphi \circ \psi)$ is the same set in the two clauses.

We postulate that the valuation function can be extended by the following recursive clauses for $\circ, \rightarrow$, and $\leftarrow$ :

$$
\begin{aligned}
v(\varphi \circ \psi)= & \left\langle\left\{z \in W^{+}: \exists x, y\left(R^{+} x y z \& x \in v^{+}(\varphi) \& y \in v^{+}(\psi)\right)\right\},\right. \\
& \left.\left\{y \in W^{-}: \forall x, z\left(\left(R_{\rightarrow}^{-} x y z \& x \in v^{+}(\varphi)\right) \Rightarrow z \in v^{-}(\psi)\right)\right\}\right\rangle, \\
v(\varphi \circ \psi)= & \left\langle\left\{z \in W^{+}: \exists x, y\left(R^{+} x y z \& x \in v^{+}(\varphi) \& y \in v^{+}(\psi)\right)\right\},\right. \\
& \left.\left\{y \in W^{-}: \forall x, z\left(\left(R_{\leftarrow}^{-} x y z \& x \in v^{+}(\varphi)\right) \Rightarrow z \in v^{-}(\psi)\right)\right\}\right\rangle,
\end{aligned}
$$




$$
\begin{aligned}
v(\varphi \rightarrow \psi)= & \left\langle\left\{y \in W^{+}: \forall x, z\left(\left(R^{+} x y z \& x \in v^{+}(\varphi)\right) \Rightarrow z \in v^{+}(\psi)\right)\right\},\right. \\
& \left.\left\{z \in W^{-}: \exists x, y\left(R_{\rightarrow}^{-} x y z \& x \in v^{-}(\varphi) \& y \in v^{-}(\psi)\right)\right\}\right\rangle, \\
v(\psi \leftarrow \varphi)= & \left\langle\left\{x \in W^{+}: \forall y, z\left(\left(R^{+} x y z \& y \in v^{+}(\varphi)\right) \Rightarrow z \in v^{+}(\psi)\right)\right\},\right. \\
& \left.\left\{z \in W^{-}: \exists x, y\left(R_{\leftarrow}^{-} x y z \& x \in v^{-}(\varphi) \& y \in v^{-}(\psi)\right)\right\}\right\rangle,
\end{aligned}
$$

where $v^{+}(\varphi * \psi)=\ell v^{-}(\varphi * \psi)$ and $v^{-}(\varphi * \psi)=r v^{+}(\varphi * \psi)$ for $* \in\{\circ, \rightarrow, \leftarrow\}$.

With the above definitions added, the augmented frame and model can serve as a basis for the definition of semantic consequence for $L S$. Since in $L S$ the structure $\Gamma$ is possibly not a singleton, we extend the definition of $\vDash$ to multiple cases. We will use grouping of arguments of $R^{+}$as usual (that is, for $R$ ), $R^{+}(x y) z v \Leftrightarrow \exists u\left(R^{+} x y u \& R^{+} u z v\right)$ and $R^{+} x(y z) v \Leftrightarrow \exists u\left(R^{+} y z u \& R^{+} x u v\right)$.

Definition 4.1 (Semantical consequence relation) Let $\Gamma$ be a finite sequence of formulas $\left(\gamma_{1}, \ldots, \gamma_{n}\right)$ with well-balanced parentheses. $\varphi$ is the semantical consequence of $\Gamma(\Gamma \vDash \varphi)$ if and only if $w^{+} \in v^{+}(\Gamma)$ implies $w^{+} \in v^{+}(\varphi)$, where

1. $v^{+}(\Gamma)=\left\{z: \exists x_{1}, \ldots, \exists x_{n} R^{+}\left(x_{1}, \ldots, x_{n}\right) z\right.$

$\left.\& \forall i\left(2 \leq n \geq i \geq 1 \Rightarrow x_{i} \in v^{+}\left(\gamma_{i}\right)\right)\right\}$, and

2. $v^{+}(\Gamma)=v^{+}\left(\gamma_{1}\right)$ otherwise.

$\Gamma$ can be equivalently thought of as an ordered binary tree, that is, if $\Gamma$ is not completely parenthesized, then the missing parentheses are to be restored as associated to the left. By the similarity of the notation for $\left(\gamma_{1}, \ldots, \gamma_{n}\right)$ and $\left(x_{1}, \ldots, x_{n}\right)$ we meant to indicate that their grouping is the same.

Now we can consider the relation between syntactical and semantical consequence. First we prove soundness.

\section{Theorem 4.2 (Soundness) $\quad$ If $\Gamma \vdash \varphi$, then $\Gamma \vDash \varphi$.}

Proof All provable sequents in $L S$ are finite, and any structures on the left that contain commas can be turned into a single formula by finitely many applications of the left introduction rule for fusion. Moreover, the $(\circ \vdash)$ rule is reversible (since cut is admissible in $L S$ ); therefore, we will treat $\Gamma$ and its fused version as the same.

As we noted already $\wedge$ and $\vee$ are dealt with as before. We first show that $\rightarrow$ is a residual of $\circ$. (The case of $\leftarrow$ is symmetric.)

(1) Let us assume that $v^{+}(\varphi \circ \psi) \subseteq v^{+}(\chi)$ and $y \in v^{+}(\psi)$. Additionally we also assume that $R^{+} x y z$ and $x \in v^{+}(\varphi)$. By the definition of $v$ for $\circ$ it follows that $z \in v^{+}(\varphi \circ \psi)$; hence by the first assumption $z \in v^{+}(\chi)$. The elimination of the additional assumptions gives $\forall x, z\left(\left(R^{+} x y z \& x \in v^{+}(\varphi)\right) \Rightarrow z \in v^{+}(\chi)\right)$, that is, the desired conclusion $y \in v^{+}(\varphi \rightarrow \chi)$ according to the definition of $v$ for $\rightarrow$.

(2) For the other direction let us assume $v^{+}(\psi) \subseteq v^{+}(\varphi \rightarrow \chi)$ and $z \in v^{+}(\varphi \circ \psi)$. Then $\exists x, y\left(R^{+} x y z \& x \in v^{+}(\varphi) \& y \in v^{+}(\psi)\right)$ by the definition of $v$ for $\circ$. By the first assumption $\exists x, y\left(R^{+} x y z \& x \in v^{+}(\varphi) \& y \in v^{+}(\varphi \rightarrow \psi)\right)$. Now we assume to the contrary that $z \notin v^{+}(\chi)$. For any $y \in v^{+}(\varphi \rightarrow \chi)$, by the definition of $v$ for $\rightarrow, \forall x^{\prime}, z^{\prime}\left(\left(R^{+} x^{\prime} y z^{\prime} \& x^{\prime} \in v^{+}(\varphi)\right) \Rightarrow z^{\prime} \in v^{+}(\chi)\right)$, that is, $\forall x^{\prime}, z^{\prime}\left(\left(z^{\prime} \notin v^{+}(\chi) \Rightarrow \neg\left(R^{+} x^{\prime} y z^{\prime} \& x^{\prime} \in v^{+}(\varphi)\right)\right)\right.$. A one-step universal instantiation and detachment leads to $\forall x^{\prime}\left(\neg R x^{\prime} y z \vee x^{\prime} \notin v^{+}(\varphi)\right)$ that contradicts $z \in v^{+}(\varphi \circ \psi)$. Therefore, $z \in v^{+}(\chi)$. 
(3) We show that $\circ$ is lattice ordered. Since $\vee$ is disjunction (as we know from Theorem 2.2), we have that $v^{+}(\varphi) \subseteq v^{+}(\varphi \vee \psi)$ and also $v^{+}(\psi) \subseteq v^{+}(\varphi \vee \psi)$. Then $v^{+}(\varphi \circ \chi) \subseteq v^{+}((\varphi \vee \psi) \circ \chi)$, and $v^{+}(\psi \circ \chi) \subseteq v^{+}((\varphi \vee \psi) \circ \chi)$. Therefore, $v^{+}((\varphi \circ \chi) \vee(\psi \circ \chi)) \subseteq v^{+}((\varphi \vee \psi) \circ \chi)$.

To show the other direction we need a slightly more subtle argument. Again using the fact that $\vee$ is disjunction we have that $v^{+}(\varphi \circ \chi) \subseteq v^{+}((\varphi \circ \chi) \vee(\psi \circ \chi))$ and $v^{+}(\psi \circ \chi) \subseteq v^{+}((\varphi \circ \chi) \vee(\psi \circ \chi))$. We have just established in steps (1) and (2) that residuation holds; therefore, we get that $v^{+}(\varphi) \subseteq v^{+}(((\varphi \circ \chi) \vee(\psi \circ \chi)) \leftarrow \chi)$ and that $v^{+}(\psi) \subseteq v^{+}(((\varphi \circ \chi) \vee(\psi \circ \chi)) \leftarrow \chi)$. Applying the semantical equivalent of disjunction introduced in the last two inclusions, we obtain $\left.v^{+}(\varphi \vee \psi) \subseteq v^{+}((\varphi \circ \chi) \vee(\psi \circ \chi)) \leftarrow \chi\right)$. Using residuation in the other direction we arrive at the desired inclusion $v^{+}((\varphi \vee \psi) \circ \chi) \subseteq v^{+}((\varphi \circ \chi) \vee(\psi \circ \chi))$. (The distribution of o over $\vee$ from the left can be shown similarly, of course.)

The canonical frame consists of two sets, the set of positive theories $\mathcal{T}^{+}$and the set of negative theories $\mathcal{T}^{-}$and the relation $\chi$ (which is nonempty intersection as before). The canonical $R^{+}, R_{\rightarrow}^{-}$, and $R_{\leftarrow}^{-}$are defined in (16) and (17) below. Note that in (16) $x, y, z$ are all members of $\mathcal{T}^{+}$, but in (17) $x \in \mathcal{T}^{+}$and $y, z \in \mathcal{T}^{-}$.

$$
\begin{aligned}
& R^{+} x y z \Longleftrightarrow \forall \varphi, \psi((\varphi \in x \& \psi \in y) \Rightarrow \varphi \circ \psi \in z), \\
& R_{\rightarrow}^{-} x y z \Longleftrightarrow \forall \varphi, \psi((\varphi \in x \& \psi \in y) \Rightarrow \varphi \rightarrow \psi \in z), \\
& R_{\leftarrow}^{-} x y z \Longleftrightarrow \forall \varphi, \psi((\varphi \in x \& \psi \in y) \Rightarrow \psi \leftarrow \varphi \in z) .
\end{aligned}
$$

The definition of $R^{+}$is just like the ordinary definition of the ternary accessibility relation in relevance logics. The definitions of $R_{\rightarrow}^{-}$and $R_{\leftarrow}^{-}$in (17) are motivated by the fact that implications are antitone in their first argument.

The canonical model is defined on the canonical frame by adding a valuation function $v$ that is defined on propositional variables as

$$
v(p)=\left\langle\left\{T^{+} \in \mathcal{T}^{+}: p \in T^{+}\right\},\left\{T^{-} \in \mathcal{T}^{-}: p \in T^{-}\right\}\right\rangle .
$$

The canonical model so defined is indeed defined on a frame with $v$ satisfying conditions (13) - (15). It also has the separation property; that is, $L S$ is complete for this semantics.

\section{Theorem 4.3 (Completeness) If $\Gamma \vDash \varphi$, then $\Gamma \vdash \varphi$.}

Proof First we note that the canonical frame is a frame, the sets of theories are not empty, and all the relations are of the required type. We prove that the canonical model is a model with the separation property.

(1) The cases of $\wedge$ and $\vee$ are dealt with as in Theorem 2.5. Thus we omit repeating the steps.

We give here two further steps of the proof that $\left\langle T^{+}, T^{-}\right\rangle \in v(\varphi)$ if and only if $\varphi \in T^{+}$and $\varphi \in T^{-}$, namely, the case for $\circ$ and $\rightarrow$. (For $\leftarrow$ the claim is proved like that for $\rightarrow$ with the obvious modification that $R_{\leftarrow}^{-}$replaces $R_{\rightarrow}^{-}$.)

(2) Assume that $T^{+} \in v^{+}(\varphi \circ \psi)$. Then, by the definition above, $\exists x, y\left(R^{+} x y T^{+} \& x \in v^{+}(\varphi) \& y \in v^{+}(\psi)\right)$. The induction hypothesis gives $\exists x, y\left(R^{+} x y T^{+} \& \varphi \in x \& \psi \in y\right)$, but this together with the canonical definition of $R^{+}$implies $\varphi \circ \psi \in T^{+}$. To prove the converse, assume that the latter holds. According to the definition of $R^{+}, R^{+}[\varphi)[\psi) T^{+}$. Thus $\exists x, y\left(R^{+} x y T^{+} \& \varphi \in x \&\right.$ 
$\psi \in y)$. Applying the inductive hypothesis we have $\exists x, y\left(R^{+} x y T^{+} \& x \in v^{+}(\varphi) \&\right.$ $\left.y \in v^{+}(\psi)\right)$; that is, $T^{+} \in v^{+}(\varphi \circ \psi)$.

Now we show that $T^{-} \in v^{-}(\varphi \circ \psi)$ if and only if $(\varphi \circ \psi) \in T^{-}$. First we show this from right to left. Assume that $\varphi \circ \psi \in T^{-}$. Additionally, let us suppose also that $R_{\rightarrow}^{-} x T^{-} z$ for some $x$ and $z$, with $\varphi \in x$. By the definition of $R_{\rightarrow}^{-}, \varphi \rightarrow(\varphi \circ \psi)$ is in $z$. However, $\psi \vdash \varphi \rightarrow(\varphi \circ \psi)$ and so $\psi \in z$ too. Using the inductive hypothesis for $\varphi \in x$ and $\psi \in z$ and eliminating the additional assumptions, we get $\forall x, z\left(\left(R_{\rightarrow}^{-} x T^{-} z \& x \in v^{+}(\varphi)\right) \Rightarrow z \in v^{-}(\psi)\right)$; that is, $T^{-} \in v^{-}(\varphi \circ \psi)$.

To prove that the inclusion holds in the other direction, assume that $(\varphi \circ \psi) \notin T^{-}$. $[\varphi)$ is a member of $v^{+}(\varphi)$ (by inductive hypothesis). Define a set of negative theories $C$ as $C=\left\{z: R_{\rightarrow}^{-}[\varphi) T^{-} z \& \psi \notin z\right\}$. $C$ is not empty (since $[\varphi)$ and $T^{-}$ are nonempty) and closed under unions of nonempty chains (with respect to $\subseteq$ as ordering). Let $z^{\prime}$ be the maximal element of $C$. From the construction it follows that $R_{\rightarrow}^{-}[\varphi) T^{-} z^{\prime}$ and $\psi \notin z^{\prime}$; that is, $\neg \forall x, z\left(\left(R_{\rightarrow}^{-} x T^{-} z \& x \in v^{+}(\varphi)\right) \Rightarrow z \in v^{-}(\psi)\right)$. Hence $T^{-} \notin v^{-}(\varphi \circ \psi)$. Showing that $T^{-} \in v^{-}(\varphi \circ \psi)$ if and only if $(\varphi \circ \psi) \in T^{-}$ holds using $R_{\leftarrow}^{-}$is similar.

(3) Now we proceed to the case of $\rightarrow$. First assume that $\varphi \rightarrow \psi \in T^{+}$. Additionally, let us suppose that $R^{+} x T^{+} z$ and $\varphi \in x$. Then $\varphi \circ(\varphi \rightarrow \psi) \in z$ by the definition of $R^{+}$. However, $\varphi \circ(\varphi \rightarrow \psi) \vdash \psi$ and so $\psi \in z$ too. By induction hypothesis $x \in v^{+}(\varphi)$ and $z \in v^{+}(\psi)$, so $\forall x, z\left(\left(R^{+} x T^{+} z \& x \in v^{+}(\varphi)\right) \Rightarrow z \in v^{+}(\psi)\right)$. This implies, by the definition of $\rightarrow, T^{+} \in v^{+}(\varphi \rightarrow \psi)$. Again we proceed by demonstrating the contrapositive of the other inclusion. Let us assume that $T^{+} \notin v^{+}(\varphi \rightarrow \psi)$. We define $C$, a set of positive theories, as $C=\left\{z: R^{+}[\varphi) T^{+} z \& \psi \notin z\right\}$. Let the maximal element of $C$ be denoted as $z^{\prime}$. Then $R^{+}[\varphi) T^{+} z^{\prime}$ and $\psi \notin z^{\prime}$. Applying the inductive hypothesis and introducing existential quantifiers, we get $\exists x, z\left(R^{+} x T^{+} z \& x \in v^{+}(\varphi) \& z \notin v^{+}(\psi)\right)$. Hence, $T^{+} \notin v^{+}(\varphi \rightarrow \psi)$ as desired.

For $\rightarrow$ the case involving negative theories is almost obvious, since we used $R_{\rightarrow}^{-}$to define the falsity conditions. Assume that $T^{-} \in v^{-}(\varphi \rightarrow \psi)$. Then $\exists x, y\left(R_{\rightarrow}^{-} x y T^{-} \& x \in v^{+}(\varphi) \& y \in v^{-}(\psi)\right)$. By induction hypothesis, $\varphi \in x$ and $\psi \in y$, and from the definition of $R_{\rightarrow}^{-}$, it is immediate that $\varphi \rightarrow \psi \in T^{-}$. For the converse, suppose that the latter is the case. Consider the positive theory generated by $\varphi$ and the negative theory generated by $\psi$. These are by inductive hypothesis in $v^{+}(\varphi)$ and $v^{-}(\psi)$, respectively. Clearly, $R_{\rightarrow}^{-}[\varphi)(\psi] T^{-}$according to the definition of $R_{\rightarrow}^{-}$. By existential generalization, $\exists x, y\left(R_{\rightarrow}^{-} x y T^{-} \& x \in v^{+}(\varphi) \& y \in v^{-}(\psi)\right)$, and so $T^{-} \in v^{-}(\varphi \rightarrow \psi)$.

The insights gained from the semantics in this section motivate a neater semantics that we describe in the next section.

\section{Generalized Kripke Semantics}

The semantics we presented in Section 4 is somewhat complicated compared to the semantics of, say, $\mathbf{K}$ (the minimal normal modal logic). Our aim now is to provide a nicer semantics for the same system.

As we saw the intensional connectives have a certain similarity to their classical cousins in the way their truth and falsity is determined. For fusion it was straightforward to define its truth, and for the implications it was clear that if the antecedent was true and the consequent was false (of course, linked via a relation) then the whole 
implication should be false. Since these connectives are related to each other through residuation, the truth of the implications was definable from the same relation $\left(R^{+}\right)$ that was used in defining the truth of fusion, and the falsity of fusion was definable from the relations needed to define the falsity of the implications. An alternative definition could simply say that, for instance, a fusion is false when it is not true, where the 'not' is to be interpreted in a similar way as we did in (3) and (4) in Section 2. Whereas this way of looking at the falsity of the connectives is perhaps less informative in some sense, it allows simplifying the definition of the frame.

Before proceeding to the formal definition of our semantics we want to provide some further motivations and intuitions. It seems natural to us to assume a "protoorder" (a transitive relation $\sqsubset$ ) on the frame and take propositions to be upwardclosed sets: a proposition should include all situations that are at least as informative as the one already known to be in the proposition. Using this relation we are able to characterize the counterpropositions: those are all the situations that are not more informative than the situations in the proposition.

Previously we used positive and negative theories as the positive and the negative halves of a situation. Here we limit our consideration to positive theories, but we assume a more specific relation (than an arbitrary $Q$ ). Thus, canonically we think of a proposition corresponding to $\varphi$ as an upward-closed set of theories, each containing $\varphi$. It is as though all these theories verify $\varphi$. At the same time we obtain, via the relation $\sqsubset$, the counterproposition induced by $\varphi$, which is characterized by another set of theories that is closed downward. Each theory in the counterproposition is contained in the (minimal) theory generated by $\varphi$. It is as though all these theories refute $\varphi .{ }^{10}$ There might be still other theories which are not in either of these two sets; they have nothing definitive to say about the sentence $\varphi$, and so they do not fall into the proposition nor do they fall into the counterproposition corresponding to $\varphi$.

As it turns out if we build the two modifications mentioned above into the definition of the frame, then we get a mathematically more elegant semantics. We call this semantics the generalized Kripke semantics. We generalize here the so-called Kripke semantics which is suitable for classical modal logics, defined on a frame $\langle W, R\rangle$ (where $R$ is an $n+1$-ary relation for an $n$-ary additive operation), to a semantics which is suitable for positive substructural logics, defined on a frame $\langle W, \sqsubset, R\rangle$ (where $\sqsubset$ is a transitive binary relation and $R$ is an $n+1$-ary relation for an $n$-place additive operation).

Earlier the definition of the two functions $\ell$ and $r$ was based on a polarity, and $Q$ was an arbitrary binary relation between the two sets $W^{+}$and $W^{-}$. The canonical valuation mapped a formula $\varphi$ into a pair of upward-closed sets, the set of positive theories containing $\varphi$ and the set of negative theories containing $\varphi$; both of these sets were upward closed with respect to set inclusion. Now we can take a slightly more specific structure than a polarity and still get a suitable construction. Namely, a transitive relation $\sqsubset$ on one set $W$ (with the same definitions of $\ell$ and $r$ as before) yields a semilattice of $\ell r$-closed subsets of $W$ and a semilattice of $r \ell$-closed subsets of $W$. Note that the two functions $\ell$ and $r$ map subsets of $W$ into upward- and downward-closed ones, respectively, (where if $X \subseteq W$ then $X$ is upward closed if and only if $x \in X$ and $x \sqsubset y$ implies $y \in X$, and $X$ is downward closed if and only if $x \in X$ and $y \sqsubset x$ implies $y \in X$ ). Lastly, instead of three accessibility relations we have just one, denoted as $R$. 
Formally, the frame is a triple $\mathcal{F}=\langle W, \sqsubset, R\rangle . W$ is a nonempty set; $\sqsubset \subseteq W \times W$, and if $x \sqsubset y$ and $y \sqsubset z$ then $x \sqsubset z ; R \subseteq W^{3}$. The frame is required to satisfy (18) that relates $\sqsubset$ and $R$ :

$$
\begin{aligned}
& \left(R x y z \& x^{\prime} \sqsubset x\right) \quad \Rightarrow \quad R x^{\prime} y z, \\
& \left(R x y z \& y^{\prime} \sqsubset y\right) \quad \Rightarrow \quad R x y^{\prime} z, \\
& \left(R x y z \& z \sqsubset z^{\prime}\right) \quad \Rightarrow \quad R x y z^{\prime} .
\end{aligned}
$$

These tonicity conditions are the usual ones except that we defined them on a protoordered set. Were we to use a quasi order $\sqsubseteq$, that is, a relation that is reflexive and transitive, instead of $\sqsubset$, the three lines in (18) could have been concisely written as

$$
\left(R x y z \& x^{\prime} \sqsubseteq x \& y^{\prime} \sqsubseteq y \& z \sqsubseteq z^{\prime}\right) \Rightarrow R x^{\prime} y^{\prime} z^{\prime} .
$$

Using a quasi order would not cause any problem in the semantics, since set theoretical inclusion is reflexive; however, reflexivity is not used anywhere in the proof of soundness.

As before, we define two functions $\ell, r$ from $\sqsubset$ :

$$
\begin{aligned}
r(X) & =\{y: \forall x(x \in X \Rightarrow y \sqsubset x)\}, \\
\ell(Y) & =\{x: \forall y(y \in Y \Rightarrow y \sqsubset x)\} .
\end{aligned}
$$

A routine verification shows that the two functions do result in downward- and upward-closed subsets as we claimed earlier. We denote the set of upward-closed subsets of a set $X$ by $\wp(X)^{\uparrow}$ and the set of $\ell r$-closed subsets of $X$ by $\wp(X)^{\ell r}$.

A model is a frame augmented with a valuation function $v$ that maps propositional variables into $\wp(W)^{\ell r}$ and can be extended to map complex formulas into $\wp(W)^{\ell r}$ according to (21) - (25).

Conjunction and disjunction are defined as

$$
\begin{aligned}
& v(\varphi \wedge \psi)=v(\varphi) \cap v(\psi), \\
& v(\varphi \vee \psi)=\ell(r v(\varphi) \cap r v(\psi)) .
\end{aligned}
$$

The intensional operations are defined as

$$
\begin{aligned}
v(\varphi \circ \psi) & =\{z: \exists x, y(\operatorname{Rxyz} \& x \in v(\varphi) \& y \in v(\psi))\}, \\
v(\varphi \rightarrow \psi) & =\{y: \forall x, z((\operatorname{Rxyz} \& x \in v(\varphi)) \Rightarrow z \in v(\psi))\}, \\
v(\psi \leftarrow \varphi) & =\{x: \forall y, z((\operatorname{Rxyz} \& y \in v(\varphi)) \Rightarrow z \in v(\psi))\} .
\end{aligned}
$$

We note that if we want to model only additive operations (like ordinary modal logic does) as, for instance, the binary o, then we also have to postulate conditions similar to the following two:

$$
\begin{aligned}
& v(\varphi \circ(\psi \vee \chi)) \subseteq v((\varphi \circ \psi) \vee(\varphi \circ \chi)), \\
& v((\psi \vee \chi) \circ \varphi) \subseteq v((\psi \circ \varphi) \vee(\chi \circ \varphi)) .
\end{aligned}
$$

We will use the notation $w \vDash \varphi$ to mean, as before, that ' $\varphi$ is at least true in the situation $w^{\prime}$. However, formally this means now that $w \in v(\varphi)$. The definition of $\Gamma \vDash \varphi$ is modified accordingly, that is, the $+\mathrm{s}$ are omitted from $v^{+} \mathrm{s}$.

Theorem 5.1 (Soundness) If $\Gamma \vdash \varphi$, then $\Gamma \vDash \varphi$.

Proof We give some details of the steps that show that residuation and distributivity of fusion over join hold. (The cases for $\wedge$ and $\vee$ are similar to the previous ones, hence omitted.) 
Case 1 Let us assume that $v(\varphi \circ \psi) \subseteq v(\chi)$ and $y \in v(\psi)$. From the former we get that if $\exists x, y(R x y z \& x \in v(\varphi) \& v(\psi))$ then $z \in v(\chi)$. If also Rxyz and $x \in v(\varphi)$, then $z \in v(\chi)$; that is, $\forall x, z((\operatorname{Rxyz} \& x \in v(\varphi)) \Rightarrow z \in v(\chi))$. Therefore, $y \in v(\varphi \rightarrow \chi)$. Thus, we have proved that if $v(\varphi \circ \psi) \subseteq v(\chi)$ then $v(\psi) \subseteq v(\varphi \rightarrow \chi)$. To prove the converse, let us assume that the latter holds and $z \in v(\varphi \circ \psi)$. The definition of $\circ$ gives $\exists x, y(\operatorname{Rxyz} \& x \in v(\varphi) \& y \in v(\psi))$. Instantiations of the universal quantifiers in the first assumption and two detachments yield $z \in v(\chi)$ as we wanted. (Showing that $\leftarrow$ is a residual of $\circ$ is completely symmetric to this case, and therefore, we omit it.)

Case 2 We want to show that $v(\varphi \circ(\psi \vee \chi))=v((\varphi \circ \psi) \vee(\varphi \circ \chi))$ as well as $v((\psi \vee \chi) \circ \varphi)=v((\psi \circ \varphi) \vee(\chi \circ \varphi))$. In (27) we postulated half of the latter equality, so we proceed to show that the other inclusion holds. (Of course, if implications are in the language, then (26) and (27) can be shown to hold as before.) Assume that for some $w_{1}$ it is the case that $\forall w_{2}\left(\exists x, y\left(R x y w_{2} \& x \in v(\psi \vee \chi) \& y \in v(\varphi)\right) \Rightarrow\right.$ $\left.w_{1} \sqsubset w_{2}\right)$. Since $\vee$ is join, we have that if $x \notin v(\psi \vee \chi)$ then $x \notin v(\psi)$ and $x \notin v(\chi)$. Thus, by first-order logic, we get from the quantified formula that $\forall w_{2}\left(\exists x, y\left(R x y w_{2} \& x \in v(\psi) \& y \in v(\varphi)\right) \Rightarrow w_{1} \sqsubset w_{2}\right)$ and $\forall w_{2}\left(\exists x, y\left(R x y w_{2} \& x \in v(\chi) \& y \in v(\varphi)\right) \Rightarrow w_{1} \sqsubset w_{2}\right)$. The definitions of $\circ, \vee$, and $r$ give that if $w_{1} \in r v((\psi \vee \chi) \circ \varphi)$ then $w_{1} \in r v((\psi \circ \varphi) \vee(\chi \circ \varphi))$. Then, by Schmidt's definition of a Galois connection, we get that $v((\psi \circ \varphi) \vee(\chi \circ \varphi)) \subseteq$ $\ell r v((\psi \vee \chi) \circ \varphi)$. Since by definition the operations result in $\ell r$-closed sets, we have that $v((\psi \circ \varphi) \vee(\chi \circ \varphi)) \subseteq((\psi \vee \chi) \circ \varphi)$, what we wanted to prove. (The case of $v(\varphi \circ(\psi \vee \chi)) \supseteq v((\varphi \circ \psi) \vee(\varphi \circ \chi))$ is just like this one, since nothing distinguishes the two argument places in $\circ$ except one being the first and the other being the second that we did not rely on in the proof.)

The canonical frame is the set $\mathcal{T}$, the set of theories, with $\subseteq$, set inclusion that is a partial order on $\mathcal{T}$, and with $R$ defined as

$$
R x y z \Longleftrightarrow \forall \varphi, \psi((\varphi \in x \& \psi \in y) \Rightarrow \varphi \circ \psi \in z) .
$$

The canonical valuation is defined in a standard way:

$$
v(p)=\{T: T \in \mathcal{T} \text { and } p \in T\} .
$$

Before we proceed to state and prove completeness we state and prove a couple of useful lemmas about the canonical frame. The first lemma says that $\ell r$-closed sets are closed under intersection. The second one connects the $\ell r$-closed and the upward-closed sets via the intersection closure.

Lemma 5.2 ( $\ell$-closed sets are $\bigcap$-closed) If $X \in \wp(\mathcal{T})^{\ell r}$, then $\bigcap X \in X$.

Proof Assume that $X \in \wp(\mathcal{T})^{\ell r}$, then $\bigcap X$ is a theory. By the properties of intersection, $(\forall T \in X)(\bigcap X \subseteq T)$. This means that $\bigcap X \in r X$. We want to show that for any $T^{\prime}$ such that $T^{\prime} \in r X, T^{\prime} \subseteq \bigcap X$. Let us assume to the contrary that $T^{\prime} \nsubseteq \bigcap X$, that is, $\left(\exists w \in T^{\prime}\right)(w \notin \bigcap X)$. Then for some $T^{\prime \prime} \in X, w \notin T^{\prime \prime}$. But then $T^{\prime} \nsubseteq T^{\prime \prime}$, and so $T^{\prime} \notin r X$, but this contradicts $T^{\prime} \in r X$. It follows then that $\bigcap X \in \ell r X$, and since $X=\ell r X$ by assumption, $\bigcap X \in X$. 
Not all upward-closed sets are $\ell r$-closed. One can easily construct an example of an upward-closed set of theories on the minimal modular lattice $M_{3}$ that is not $\ell r$ closed. The next lemma grasps the characteristic feature of $\ell r$-closed sets among the upward-closed ones using $\bigcap$-closure.

Lemma 5.3 ( $\bigcap$-closure is characteristic) If $X \in \wp(\mathcal{T})^{\uparrow}$, then $X \in \wp(\mathcal{T})^{\ell r}$ if and only if $\bigcap X \in X$.

Proof From left to right it is a corollary of Lemma 5.2. For the other direction assume that $X \in \wp(\mathcal{T})^{\uparrow}$ and $\bigcap X \in X$. If $w_{2} \in \ell r X$, then $\forall w_{1}\left(w_{1} \in r X \Rightarrow w_{1} \subseteq w_{2}\right)$; that is, for any $w_{3} \in X, w_{1} \subseteq w_{3}$. But this means that $w_{1} \subseteq \bigcap X$. Since $\forall w_{3}\left(w_{3} \in X \Rightarrow \bigcap X \subseteq w_{3}\right), w_{2} \in X . X \subseteq \ell r X$ since $\ell r$ is a closure operator, and so indeed, $X=\ell r X$; that is, $X \in \wp(\mathcal{T})^{\ell r}$.

Now we are ready for the completeness theorem.

Theorem 5.4 (Completeness) If $\Gamma \vDash \varphi$, then $\Gamma \vdash \varphi$.

Proof First we show that the canonical frame is a frame. Furthermore, we prove that any valuation that maps the propositional variables into $\ell r$-closed subsets of $\mathcal{T}$ can be extended to a valuation for all formulas (mapping them into $\ell r$-closed subsets of $\mathcal{T}$ ); moreover, residuation, and so also distributivity (of $\circ$ over $\vee$ ), holds. We also prove that the latter property holds even if the implications are not in the language of the logic. (In the modal logic literature, a similar feature of the canonical frame is called canonicity.) The conditions needed to ensure that $\circ$ distributes over $\vee$ from both sides without $\rightarrow$ and $\leftarrow$ present are not first-order sentences in the meta-language. Thus, it is not at all obvious that the conditions hold on the canonical frame. Next we prove that $\varphi \in T$ if and only if $T \in v(\varphi)$ for arbitrary formulas (where $v$ is the canonical valuation). Lastly, we show that the canonical valuation provides separation.

(1) Clearly, $\mathcal{T}$ is a nonempty set, $\subseteq$ is a transitive relation (even more, a partial order) on $\mathcal{T}$, and $R$ is a relation on $\mathcal{T}^{3}$. The only thing to prove is that $\subseteq$ and $R$ are related in the desired way. For instance, the first clause from (18) can be shown to hold as follows. Assume Rxyz and $x^{\prime} \subseteq x$. If $a \in x^{\prime}$, then $a \in x$. Since $\forall a, b((a \in x \& b \in y) \Rightarrow a \circ b \in z)$, it is immediate that $\forall a, b\left(\left(a \in x^{\prime} \& b \in y\right) \Rightarrow a \circ b \in z\right)$ which means exactly what is wanted: $R x^{\prime} y z$. (The two other cases are similar and so omitted.) This completes the proof that the canonical frame is a frame.

(2) Now we show that any valuation that assigns to propositional variables members of $\wp(\mathcal{T})^{\ell r}$ is such that when extended according to the definitions (21) - (25) yields $\ell r$-closed subsets of $\mathcal{T}$.

(2.1) Let us assume that $v(\varphi), v(\psi) \in \wp(\mathcal{T})^{\ell r}$. If $\bigcap v(\varphi \wedge \psi) \notin v(\varphi \wedge \psi)$ then there are $x_{1}, x_{2} \in v(\varphi \wedge \psi)$ such that $x_{1} \cap x_{2} \notin v(\varphi \wedge \psi)$. From the definition of $\wedge$ we get that $x_{1} \in v(\varphi) \cap v(\psi)$ and $x_{2} \in v(\varphi) \cap v(\psi)$. But then $x_{1}, x_{2} \in v(\varphi)$ and so $x_{1} \cap x_{2} \in v(\varphi \wedge \psi)$, contrary to our assumption. Since $v(\varphi)$ and $v(\psi)$ are both upward-closed sets, so is their intersection; that is, $v(\varphi \wedge \psi) \in \wp(\mathcal{T})^{\uparrow}$, and by Lemma 5.3, $v(\varphi \wedge \psi) \in \wp(\mathcal{T})^{\ell r}$.

Let us assume once again that $v(\varphi), v(\psi) \in \wp(\mathcal{T})^{\ell r}$ and that $\bigcap v(\varphi \vee \psi) \notin$ $v(\varphi \vee \psi)$. Then there are $x_{1}, x_{2} \in v(\varphi \vee \psi)$ such that $x_{1} \cap x_{2} \notin v(\varphi \vee \psi)$. By the definition of $\vee, \forall y\left((y \in r v(\varphi) \cap r v(\psi)) \Rightarrow y \subseteq x_{1}\right)$, and similarly for $x_{2}$. However, 
any such $y \in r v(\varphi)$ is such that $y \subseteq \bigcap v(\varphi)$, and for $y \in r v(\psi) \quad y \subseteq \bigcap v(\psi)$. It follows then that $\bigcap v(\varphi) \subseteq x_{1}$ and $\bigcap v(\varphi) \subseteq x_{2}$ and so, by properties of intersection, $\bigcap(\varphi) \subseteq x_{1} \cap x_{2}$. The argument repeated for $\bigcap v(\psi)$ yields that $\bigcap v(\psi) \subseteq x_{1} \cap x_{2}$, and therefore, $x_{1} \cap x_{2} \in \ell(r v(\varphi) \cap r v(\psi))$, contrary to the earlier assumption. Thus $\bigcap v(\varphi \vee \psi) \in v(\varphi \vee \psi) . v(\varphi \vee \psi)$ is upward closed, since its definition is of the form $\ell(X)$; by Lemma 5.3, $v(\varphi \vee \psi) \in \wp(\mathcal{T})^{\ell r}$.

Let us assume that $v(\varphi), v(\psi) \in \wp(\mathcal{T})^{\ell r}$. By Lemma 5.2, $\bigcap v(\varphi) \in v(\varphi)$ and $\bigcap v(\psi) \in v(\psi)$. First note that $v(\varphi \circ \psi) \in \wp(\mathcal{T})^{\uparrow}$, since $R \downarrow \uparrow$, and if $z \in v(\varphi \circ \psi)$, so are all theories that include $z$. Now assume to the contrary that $v(\varphi \circ \psi) \notin \wp(\mathcal{T})^{\ell r}$. Then by Lemma 5.3 and the fact we just noted, $\bigcap v(\varphi \circ \psi) \notin v(\varphi \circ \psi)$. Then there are some theories $z_{1}, z_{2} \in v(\varphi \circ \psi)$ such that $z_{1} \cap z_{2} \notin v(\varphi \circ \psi)$. (Of course, $z_{1} \nsubseteq z_{2}$ and $z_{2} \not \subseteq z_{1}$.) Then $\operatorname{Rxy}\left(z_{1} \cap z_{2}\right)$ does not hold for any $x \in v(\varphi)$ and $y \in v(\psi)$; therefore, $\neg R(\bigcap v(\varphi))(\bigcap v(\psi))\left(z_{1} \cap z_{2}\right)$ either. From the definition of $R$ we get that there must be an $a \in \bigcap v(\varphi)$ and a $b \in \bigcap v(\psi)$ such that $a \circ b \notin z_{1} \cap z_{2}$. However, since $z_{1}$ and $z_{2}$ are in $v(\varphi \circ \psi)$ there are some $x_{1}, x_{2} \in v(\varphi)$ and $y_{1}, y_{2} \in v(\psi)$ such that $R x_{1} y_{1} z_{1}$ and $R x_{2} y_{2} z_{2}$. Since $\bigcap$ is intersection, $a \in x_{1}$ and $a \in x_{2}$ as well as $b \in y_{1}$ and $b \in y_{2}$. From the definition of $R$ it follows that $a \circ b \in z_{1}, z_{2}$ and so in $z_{1} \cap z_{2}$, contradicting our assumption. Thus $\bigcap v(\varphi \circ \psi) \in v(\varphi \circ \psi)$, and so we have that $v(\varphi \circ \psi) \in \wp(\mathcal{T})^{\ell r}$.

The cases for the two arrows are symmetrical, thus, we go through the details of one of them. Let us assume that $v(\varphi), v(\psi) \in \wp(\mathcal{T})^{\ell r}$, but $v(\varphi \rightarrow \psi) \notin \wp(\mathcal{T})^{\ell r}$. Then $\bigcap v(\varphi \rightarrow \psi) \notin v(\varphi \rightarrow \psi)$, since $v(\varphi \rightarrow \psi) \in \wp(\mathcal{T})^{\uparrow}$. Then for some $y_{1}$ and $y_{2} \quad y_{1}, y_{2} \in v(\varphi \rightarrow \psi)$ but $y_{1} \cap y_{2} \notin v(\varphi \rightarrow \psi)$. By the definition of $\rightarrow$ there are $x$ and $z$ such that $x \in v(\varphi), R x\left(y_{1} \cap y_{2}\right) z$ but $z \notin v(\psi)$. For arbitrary $b_{1} \in y_{1}, b_{2} \in y_{2}$ we have that $b_{1} \vee b_{2} \in y_{1} \cap y_{2}$; moreover, taking an arbitrary $a \in x$, $a \circ\left(b_{1} \vee b_{2}\right) \in z$ because of the definition of $R$. Let us consider two theories $z_{1}$ and $z_{2}$, so that $R x y_{1} z_{1}$ and $R x y_{2} z_{2}$. Since $x$ and the $y$ s are nonempty, such $z$ s exist, and $a \circ b_{1} \in z_{1}$ and $a \circ b_{2} \in z_{2}$ by the definition of $R$. However, since $y_{1}, y_{2}$ are members of $v(\varphi \rightarrow \psi)$, so by universal instantiation $z_{1} \in v(\psi)$ and $z_{2} \in v(\psi)$. We also have from the original assumption $v(\psi) \in \wp(\mathcal{T})^{\ell r}$ that $\bigcap v(\psi) \in v(\psi)$ and, in particular, $z_{1} \cap z_{2} \in v(\varphi \rightarrow \psi)$. But $\left(a \circ b_{1}\right) \vee\left(a \circ b_{2}\right) \in z_{1} \cap z_{2}$; that is, $a \circ\left(b_{1} \vee b_{2}\right) \in z_{1} \circ z_{2}$. This contradicts $y_{1} \cap y_{2} \notin v(\varphi \rightarrow \psi)$ that we deduced from the assumption that $v(\varphi \rightarrow \psi) \notin \wp(\mathcal{T})^{l r}$. Thus we can conclude that $\bigcap v(\varphi \rightarrow \psi) \in v(\varphi \rightarrow \psi)$ and also $v(\varphi \rightarrow \psi) \in \wp(\mathcal{T})^{\ell r}$.

(2.2) Now we want to prove that a valuation on the canonical frame extended according to (21) $-(25)$ also satisfies residuation and distributivity of $\circ$ over $\vee$. To prove the former we refer back to the first part of the proof of Theorem 4.2; a brief inspection of Cases (1) and (2) clarifies that the steps depended only on $R^{+}$and the definition of the first member of the pairs that $v$ assigned to a formula. Thus, they could be repeated here with a few notational changes such as omitting ${ }^{+} \mathrm{s}$.

(2.3) Now we consider the case when $\circ$ is the only connective, and hence (26) (27) must be shown to hold. (We already showed that the converse directions of these inclusions always hold.) Let us assume that $v(\varphi), v(\psi) \in \wp(\mathcal{T})^{\ell r}$ and $z \in v(\varphi \circ(\psi \vee \chi))$. By the definition of $R, \exists x, y(R x y z \& x \in v(\varphi) \& y \in v(\psi \vee \chi))$. First, if $y \in v(\psi)$ or $y \in v(\chi)$, then we have that $z \in v(\varphi \circ \psi)$ or $z \in v(\varphi \circ \chi)$, and so certainly $z \in v((\varphi \circ \psi) \vee(\varphi \circ \chi))$. That is, the interesting case is when neither $y \in v(\psi)$ nor $y \in v(\chi)$. From the tonicity conditions on $R(R \downarrow \uparrow \uparrow)$ it 
follows that $R(\bigcap v(\varphi))(\bigcap v(\psi \vee \chi)) z$. Now let us assume that for some $w$ we have $w \in r v(\varphi \circ \psi)$ and also $w \in r v(\varphi \circ \chi)$. By the definition of $r$, this is the case when $\forall u(u \in v(\varphi \circ \psi) \Rightarrow w \subseteq u)$ and $\forall u(u \in v(\varphi \circ \chi) \Rightarrow w \subseteq u)$. That is, $\forall u\left(\exists x^{\prime}, y^{\prime}\left(R x^{\prime} y^{\prime} u \& x^{\prime} \in v(\varphi) \& y^{\prime} \in v(\psi)\right) \Rightarrow w \subseteq u\right)$ and $\forall u\left(\exists x^{\prime}, y^{\prime}\left(R x^{\prime} y^{\prime} u \&\right.\right.$ $\left.\left.x^{\prime} \in v(\varphi) \& y^{\prime} \in v(\chi)\right) \Rightarrow w \subseteq u\right)$. The tonicity of $R$ together with the fact that we deal with $\ell r$-closed sets gives that $\forall u(R(\bigcap v(\varphi))(\bigcap v(\psi)) u \Rightarrow w \subseteq u)$ and $\forall u(R(\bigcap v(\varphi))(\bigcap v(\chi)) u \Rightarrow w \subseteq u)$. Taking arbitrary $a, b_{1}, b_{2}$ such that $a \in \bigcap v(\varphi), b_{1} \in \bigcap v(\psi)$, and $b_{2} \in \bigcap v(\chi), R(\bigcap v(\varphi))(\bigcap v(\psi)) u$ yields $a \circ b_{1} \in u$, and so does $R(\bigcap v(\varphi))(\bigcap v(\chi)) u \quad a \circ b_{2} \in u$. That is, for any such $a, b_{1}, b_{2}$ we have that $w \subseteq\left[\left(a \circ b_{1}\right) \vee\left(a \circ b_{2}\right)\right)$. However, for $b_{1} \in \bigcap v(\psi)$ and $b_{2} \in \bigcap v(\chi), b_{1} \vee b_{2} \in \bigcap v(\psi \vee \chi)$. Thus, from $R(\bigcap v(\varphi))(\bigcap v(\psi \vee \chi)) z$ it follows that $a \circ\left(b_{1} \vee b_{2}\right) \in z$. Since $\circ$ distributes over $\vee$ in the algebra of the logic, $\left(a \circ b_{1}\right) \vee\left(a \circ b_{2}\right)=a \circ\left(b_{1} \vee b_{2}\right)$; that is, $\left(a \circ b_{1}\right) \vee\left(a \circ b_{2}\right) \in z$. By eliminating our assumptions $\forall w((w \in r v(\varphi \circ \psi) \& w \in r v(\varphi \circ \chi)) \Rightarrow w \subseteq z)$; that is, $z \in \ell(r v(\varphi \circ \psi) \cap r v(\varphi \circ \chi))$, and by the definition of $\vee$ this means that $z \in v((\varphi \circ \psi) \vee(\varphi \circ \chi))$ as desired. (The case for the other direction is, of course, similar.)

(3) Now we show that the canonical valuation is such that $T \in v(\varphi)$ if and only if $\varphi \in T$, for an arbitrary formula $\varphi$. The case for $\wedge$ can be straightforwardly extracted from (2) in the proof of Theorem 2.5 (essentially by erasing the ${ }^{+} \mathrm{s}$ ).

To prove the claim for $\vee$, let us assume that $T \in v(\varphi \vee \psi)$. Then by definition $T \in \ell(r v(\varphi) \cap r v(\psi))$; that is, by the definition of $\ell, \forall x((x \in r v(\varphi) \cap r v(\psi)) \Rightarrow$ $x \subseteq T) . x \in r v(\varphi) \cap r v(\psi)$ if and only if $x \in r v(\varphi)$ and $x \in r v(\psi)$, and further, if and only if $\forall y(y \in v(\varphi) \Rightarrow y \subseteq x)$ and $\forall y(y \in v(\psi) \Rightarrow y \subseteq x)$. By inductive hypothesis $\forall y(\varphi \in y \Rightarrow y \subseteq x)$ and $\forall y(\psi \in y \Rightarrow y \subseteq x)$. Thus we have that $\forall x((\forall y(\varphi \in y \Rightarrow x \subseteq y) \& \forall y(\psi \in y \Rightarrow x \subseteq y)) \Rightarrow x \subseteq T)$. But then $\forall x((x \subseteq[\varphi) \& x \subseteq[\psi)) \Rightarrow x \subseteq T)$; that is, $[\varphi \vee \psi) \subseteq T$, and this is exactly when $\varphi \vee \psi \in T$. For the proof of the converse it is sufficient to note that all the moves were based on equivalences (iffs).

The case of $\circ$ falls into two parts; for the first, assume that $T \in v(\varphi \circ \psi)$. The definition of $\circ$ means that for some $x \in v(\varphi)$ and $y \in v(\psi), R x y T$. By inductive hypothesis, we get that $\varphi \in x$ and $\psi \in y$, but then the definition of $R$ provides $\varphi \circ \psi$ as desired. For the second part, let us assume that $\varphi \circ \psi \in T$. Let us take two theories $[\varphi)$ and $[\psi)$. Certainly, $R[\varphi)[\psi) T$ by the definition of $R$ and the isotonicity of $\circ$. The inductive hypothesis gives that $[\varphi) \in v(\varphi)$ and $[\psi) \in v(\psi)$. By existential generalization $\exists x, y(\operatorname{Rxy} T \& x \in v(\varphi) \& y \in v(\psi))$, that is, $T \in v(\varphi \circ \psi)$ by the definition of $\circ$.

We show now that $T \in v(\psi \leftarrow \varphi)$ if and only if $\psi \leftarrow \varphi \in T$. Let us first assume that $T \in v(\psi \leftarrow \varphi)$ and that $\psi \leftarrow \varphi \notin T$-contrary to what we are trying to show. Let $C$ be defined as $C=\{z: R T[\varphi) z \& \psi \notin z\}$ (where $z \in \mathcal{T}$, of course). Taking the maximal element of $C$, we get a theory that does not contain $\psi$. But by inductive hypothesis $[\varphi) \in v(\varphi)$ and $z \notin v(\psi)$. Hence, introducing an existential quantifier, we get $\exists y, z(R T y z \& y \in v(\varphi) \& z \notin v(\psi))$. Applying the definition of $\leftarrow$ we arrive at $T \notin v(\psi \leftarrow \varphi)$. Then we may conclude that $\psi \leftarrow \varphi \in T$ as we aimed to show. For the converse, assume that $\psi \leftarrow \varphi \in T$. Let us additionally assume also that $R T y z$ for some $y$ such that $\varphi \in y$. Since $\psi \leftarrow \varphi \in T$ and $\varphi \in y$, it follows by the definition of $R$ that $(\psi \leftarrow \varphi) \circ \varphi \in z$. However, then $\psi \in z$ since $(\psi \leftarrow \varphi) \circ \varphi \vdash \psi$. 
By inductive hypothesis, $y \in v(\varphi)$ and $z \in v(\psi)$. The elimination of the additional assumptions gives that $\forall y, z((R T y z \& y \in v(\varphi)) \Rightarrow z \in v(\psi))$, that is, $T \in v(\psi \leftarrow \varphi)$ as desired. (The case of the other implication is symmetrical to this case and so omitted.)

(4) To finish the proof of completeness it remains to be shown that if $\Gamma \nvdash \varphi$ then $\Gamma \not \models \varphi$. The theory $[\varphi)$ is, of course, in $v(\varphi)$. Taking the theory generated by the fusion of $\Gamma$ it does not contain $\varphi$, since $\Gamma \nvdash \varphi$. Thus, this theory is not in $v(\varphi)$; however, this is in $v(\Gamma)$. Hence, $\Gamma \not \models \varphi$.

The semantics we provided greatly extends the range of logics which can be given a generalized four-valued semantics. We devoted this paper to considerations concerning the minimal nondistributive and the minimal positive substructural logic and gave proofs of soundness and completeness. In another paper we will provide four-valued semantics for further substructural logics.

\section{Notes}

. Sequent calculi were invented by Gentzen [11]. We assume familiarity with the usual notational conventions and definitions.

2. $\mathcal{F}$ is a polarity in the sense of [5]. The semantics we provide here was influenced by several other semantics for various logics, as Birkhoff and Frink [6], [9], Urquhart [19], Goldblatt [12], Allwein and Dunn [1], Hartonas and Dunn [13], and [4].

3. Maximally disjoint theory-countertheory pairs were used in Dunn [10] to provide semantics for the positive normal modal logic $\mathbf{K}_{+}$.

4. For technical reasons we allow nonproper inherent theories. Prime theories, as a rule, are defined to be proper. Note also that we did not exclude the empty set from among theories.

5. Mathematically these are decorated trees, where the edges are labeled with one of two labels and each level has exactly two forks with the same label.

6. Although we have not defined formally a measure to compare the two formalisms, it seems to us that searching for a tableau proof is simpler than searching for a proof in $L N$, since only two trees need to be constructed and then the covering should be checked. On the other hand each (attempted) proof of $\varphi \vdash \psi$ in $L N$ also records the order of the application of rules to $\varphi$ and $\psi$, a piece of information that is irrelevant to the provability of $\psi$ from $\varphi$.

$\varphi \dashv \vdash \psi$ abbreviates $\varphi \vdash \psi$ and $\psi \vdash \varphi$.

8. "Half" of distributivity $\left(v^{+}((\varphi \circ \psi) \vee(\varphi \circ \chi)) \subseteq v^{+}(\varphi \circ(\psi \vee \chi))\right)$ is true for any isotone operation when $\vee$ is join in a lattice. (Similarly, $v^{+}((\varphi \circ \chi) \vee(\psi \circ \chi)) \subseteq v^{+}((\varphi \vee \psi) \circ \chi)$ holds too.) The other "half" is true due to residuation; that is, without the implications we would need to postulate it (as well as, $v^{+}((\varphi \vee \psi) \circ \chi) \subseteq v^{+}((\varphi \circ \chi) \vee(\psi \circ \chi))$ ). Since $\circ$ can be viewed as a "relational generalization" of conjunction, it should not be surprising that the equivalent of distributivity $(a \wedge(b \vee c) \leq(a \wedge b) \vee(a \wedge c))$ does not hold in all possible cases. 
9. In fact, the definition of conjunction specified the falsity condition, and the definition of disjunction specified the truth condition only via the Galois connection, so resorting to a similar type of definition in the case of the intensional connectives is not too different.

10. We think of the minimal theory generated by $\varphi$ as the "dividing line" between those theories that verify $\varphi$ and more and those that verify $\varphi$ or less; hence, this theory ends up also among the refuting theories.

\section{References}

[1] Allwein, G., and J. M. Dunn, "Kripke models for linear logic," The Journal of Symbolic Logic, vol. 58 (1993), pp. 514-45. Zbl 0795.03013. MR 94g:03055. 190

[2] Belnap, N., "Life in the undistributed middle," pp. 31-41 in Substructural Logics, edited by K. Došen and P. Schroeder-Heister, Oxford University Press, New York, 1993. Zbl 0941.03519. MR 95m:03050. 172, 179

[3] Belnap, N. D., Jr., "A useful four-valued logic," pp. 5-37 in Modern Uses of Multiple-Valued Logic, edited by J. M. Dunn and G. Epstein, Reidel, Dordrecht, 1977. Zbl 0424.03012. MR 58:5021. 172

[4] Bimbó, K., "Semantics for structurally free logics LC+," Logic Journal of the IGPL, vol. 9 (2001), pp. 525-39. Zbl 0986.03019. MR 2002g:03042. 180, 190

[5] Birkhoff, G., Lattice Theory, American Mathematical Society, Providence, 1967. Zbl 0153.02501. MR 37:2638. 173, 175, 190

[6] Birkhoff, G., and O. Frink, Jr., "Representations of lattices by sets," Transactions of the American Mathematical Society, vol. 64 (1948), pp. 299-316. Zbl 0032.00504. MR 10,279g. 190

[7] Curry, H. B., Foundations of Mathematical Logic, McGraw-Hill Book Company, New York, 1963. Zbl 0163.24209. MR 26:6036. 180

[8] Dunn, J. M., "Relevance logic and entailment," pp. 117-229 in Handbook of Philosophical Logic, 2d edition, edited by D. M. Gabbay and F. Guenthner, Kluwer Academic Publishers, Dordrecht, 2001. Zbl 0875.03051. MR 1885182. 172, 179

[9] Dunn, J. M., "Intuitive semantics for first-degree entailments and 'coupled trees'," Philosophical Studies, vol. 29 (1976), pp. 149-68. MR 58:10311. 172, 176, 190

[10] Dunn, J. M., "Positive modal logic," Studia Logica, vol. 55 (1995), pp. 301-17. Zbl 0831.03007. MR 96f:03013. 190

[11] Gentzen, G., Untersuchungen über das logische Schließen, Wissenschaftliche Buchgesellschaft, Darmstadt, 1969. Zbl 0010.14501. MR 50:4228. 190

[12] Goldblatt, R., "Varieties of complex algebras," Annals of Pure and Applied Logic, vol. 44 (1989), pp. 173-242. Zbl 0722.08005. MR 91d:08005. 190

[13] Hartonas, C., and J. M. Dunn, "Stone duality for lattices," Algebra Universalis, vol. 37 (1997), pp. 391-401. Zbl 0902.06008. MR 98m:06012. 190

[14] Jónsson, B., and A. Tarski, "Boolean algebras with operators. I," American Journal of Mathematics, vol. 73 (1951), pp. 891-939. Zbl 0045.31505. MR 13,426c. 180 
[15] Jónsson, B., and A. Tarski, "Boolean algebras with operators. II," American Journal of Mathematics, vol. 74 (1952), pp. 127-62. Zbl 0045.31601. MR 13,524g. 180

[16] Meyer, R. K., and R. Routley, “Algebraic analysis of entailment. I,” Logique et Analyse (Nouvelle Série), vol. 15 (1972), pp. 407-28. Zbl 0336.02020. MR 48:5857. 180

[17] Routley, R., V. Plumwood, R. K. Meyer, and R. T. Brady, Relevant Logics and Their Rivals. Part I, Ridgeview Publishing Company, Atascadero, 1982. Zbl 0579.03011. MR 85k:03013. 179

[18] Smullyan, R. M., First-Order Logic, Springer-Verlag, New York, 1968. Zbl 0172.28901. MR 39:5311. 176

[19] Urquhart, A., "A topological representation theory for lattices," Algebra Universalis, vol. 8 (1978), pp. 45-58. Zbl 0382.06010. MR 56:8447. 190

kbimbo@linuxmail.org

School of Informatics

Indiana University

901 East 10th Street

Bloomington IN 47408-3912

dunn@indiana.edu 